\title{
Institutionalizing Leadership Management and Governance for Health System Strengthening in Emerging Economies: Evidence from the Partnership for Health System Strengthening in Africa (PHSSA)Programme
}

\author{
Jennifer Chepkorir, Naphtali Agata, Nicholas Kiambi, and Brenda Nangehe
}

\section{ABSTRACT}

Health systems in an emerging economy, specifically Sub-Saharan Africa (SSA) are characterized as fragile with low implementation of Universal Health Coverage. While acknowledging that the cause of the inadequacy in emerging economies is multi-factorial, other arguments are that the root cause is inadequate political and technical leadership. Evidence reveals that visionary, imaginative, decisive, responsible, and responsive leadership is insufficient to persuade all stakeholders in low-income nations in SubSaharan Africa to work together to attain the constructive goal of universal coverage. On the contrary, other academics suggest that successful leadership would establish a clear national vision for universal coverage and a commitment to achieving that objective over time. These contrasting observations motivated an interrogation of the link between health system governance and Universal Health Coverage in an emerging economy taking evidence from the PHSSA programme. Through a meta-analysis of the existing literature as well as analysis of the findings from the programme, the paper explores experiences, critical success factors and recommendations for improvement of UHC through institutionalizing health system governance in an emerging economy. The research provides evidence that the governance linkages in health systems and the outcomes they produce are contingent rather than assured, due to the variety and complexity inherent in the health system governance paradigm. The situation-specific setting of a country's health system determines what can be accomplished through health governance strategy design and implementation efforts. The paper recommends a need to create a conducive environment for adoption of health systems programmes by contextualizing health governance with regard to the larger set of governance institutions that surround it. A competency framework should also be adopted in recruitment of competent health managers. The study also recommends a need for the countries in seeking to institutionalize health system governance to develop and support an organizational structure and context that sustains leadership practices through advocacy, create an enabling environment for health systems leadership, management and governance through the development of ethics and other competences specific to universal health care situations as well as provide proper financial support system so that institutionalization of leadership, management and governance can have maximum impact on the effectiveness and efficiency of health systems. There is also a need to institutionalize short courses, seminars and conferences in health leadership, management, and governance so as to entrench participatory leadership in health systems.

Keywords: Emerging Economies, Governance, Leadership, Management, Universal Health Coverage.

\section{BACKGROUND OF THE STUDY}

Global estimations indicate that close to 150 million people finance their health with out-of-pocket expenditure of which close to 100 million people worldwide are pushed
Submitted : October 05, 2021

Published : November 15, 2021

ISSN: 2507-1076

DOI: $10.24018 /$ ejbmr.2021.6.6.1132

Jennifer Chepkorir

(e-mail: jnymamwa@ gmail.com)

Naphtali Agata

(e-mail: nagata01@ymail.com)

Nicholas Kiambi

(e-mail: Nicholas.Kiambi@ Amref.org)

Brenda Nangehe

(e-mail: Brenda.Nangehe@ amref.org)

*Corresponding Author into poverty as a result of high health expenditures (Knickman, \& Kovner, 2015). As a result, UHC is being touted as a panacea. However, healthy systems in SSA remain fragile. While conceding that the reasons for the poor implementation of universal coverage in emerging 
economies are complex, others argue that the core cause is a lack of political and technical leadership (Harries et al. 2009). National health systems in various low- and middleincome countries (LMICs) have been underperforming due to recurring difficulties both within and outside the health sector, particularly affecting the delivery of accessible and cheap healthcare services (Singh \& Sachs, 2013).

Universal Health Coverage (UHC) in Sub Saharan Africa (SSA) has received a renewed form of attention in recent years (Kieny \& Evans, 2013) and the countries continue to aggressively adopt it (WHO, 2010). However, the health system's functional capacities in these settings have increasingly decreased as a result of a failure to recognize and optimize the efforts of all organizations, institutions, structures, and resources primarily dedicated to promoting health (WHO, 2016). The role of health system governance is, thus, largely ignored and achievement of UHC remains a mirage in the emerging context.

While UHC has increasingly been embraced across the world (Lagomarsino et al. 2012), its achievement has not gone without its share of challenges mainly because of governance and leadership issues as is the case for Bangladesh and Thailand (Adams et al. 2018; Cutler, 2012) and lack of coordination in Pakistan (Zaidi et al. 2014). However, in Turkey, leadership and enforced prioritization of the program has led to its success (WHO, 2016). In SSA, all key health indicators are at worse levels than in any other emerging regions (Gonani et al. 2015). The issue is obviously multifaceted, and numerous remedies have been proposed, including the importance of strong and effective leadership and governance.

There is a lack of creative, innovative, decisive, responsible and responsive leadership, as well as transparent, exemplary, and inspirational leadership, to persuade all stakeholders to collaborate to accomplish the constructive goal of universal coverage in low-income nations (Gonani et al. 2015). Regardless of the current state of these countries' health systems and resources, competent leadership would develop a clear national goal for universal coverage and commit to achieving it gradually over time, so making progress that could be measured. While bureaucratic consensus, plans, processes, and meetings characterize health leadership and delivery in many countries, not enough attention is given on analyzing the impact of all these activities on deliverables for the people in need (Chigudu et al. 2018).

There is inadequate visionary, innovative, decisive, responsible, and responsive, transparent, exemplary, and inspirational leadership to influence all stakeholders to work together to achieve the constructive purpose of universal coverage in low-income countries (Gonani et al. 2015). Regardless of the existing status of health systems and resources in these countries, effective leadership would form a clear national vision towards universal coverage and commit to meeting this vision gradually over time thereby measurably moving towards achieving it. While health leadership and delivery in these countries is characterized by bureaucratic consensus, plans, processes, and meetings to develop systems and structures to enable processes such as recruitment, training, procurement and monitoring to take place, not enough emphasis is placed on assessing the impact of all these activities on deliverables for the people in need (Chigudu et al. 2018).

Individual leaders exert power in authoritarian ways in many African health systems, which contributes to centralized and individualized decision making and poses challenges to establishing participative leadership (Alliance for Health Policy System, 2016). The possible explanation for this state of affairs is that health managers are frequently not recruited based on competency through transparent selection but rather through political appointment, resulting in situations where whenever a person in charge of health is removed or the government changes, all managerial positions change, jeopardizing continuity and amplifying inefficiencies (WHO, 2007). Limited administrative ability has also been identified as a major contributor to most health-care inefficiencies, and it is seen as a "binding restriction" to enhancing health-care delivery (WHO, 2007). This needs the institutionalization of leadership, management, and governance in order to build effective health systems through policies, structures, and organizational culture.

Leadership, management, and governance can improve the quality of care under universal health care. Quality of healthcare can be characterized by effectiveness, efficiency, safety and patient-centeredness (Unger et al. 2003). Patientcenteredness include giving information, shared decision making as well as integrated and comprehensive care for addressing the needs for curative care, prevention, and health promotion (WHO, 2008). In the year 2001 and 2010, Amref Health Africa in response to the health care challenges in Africa, commissioned a Training Needs Assessment (TNA) study in SSA (Amref Health Africa, 2011) which established the need for leadership and governance strengthening. Therefore, Amref Health Africa together with the Ministry of Health (Kenya) and the Japan International Corporation Agency (JICA) implemented the Partnership for health system strengthening in Africa (PHSSA) capacity building programme. PHSSA is a capacity building programme on leadership, management, and governance for strengthening health systems (LMGHSS) which has been implemented in two phases between 2011-2016 and between 2016-2021. The programme focus was on strengthening leadership, management and governance for health systems strengthening in SSA through development, implementation, and dissemination of standardized training materials. The programme empowers leaders, managers, and teams to meet and master their most pressing challenges in healthcare through a conceptual model as summarized in Fig. 1.

The PHSSA: -LMG-HSS program encourages people and teams to improve their performance through participatory procedures and gender-aware approaches, allowing health leaders and policymakers to solve their own problems and achieve results. For better health gains, it develops and implements evidence-based approaches, as well as leveraging partnerships through public and private investments in leadership, management, and governance. 
People and Teams empowered to lead, manage and govern
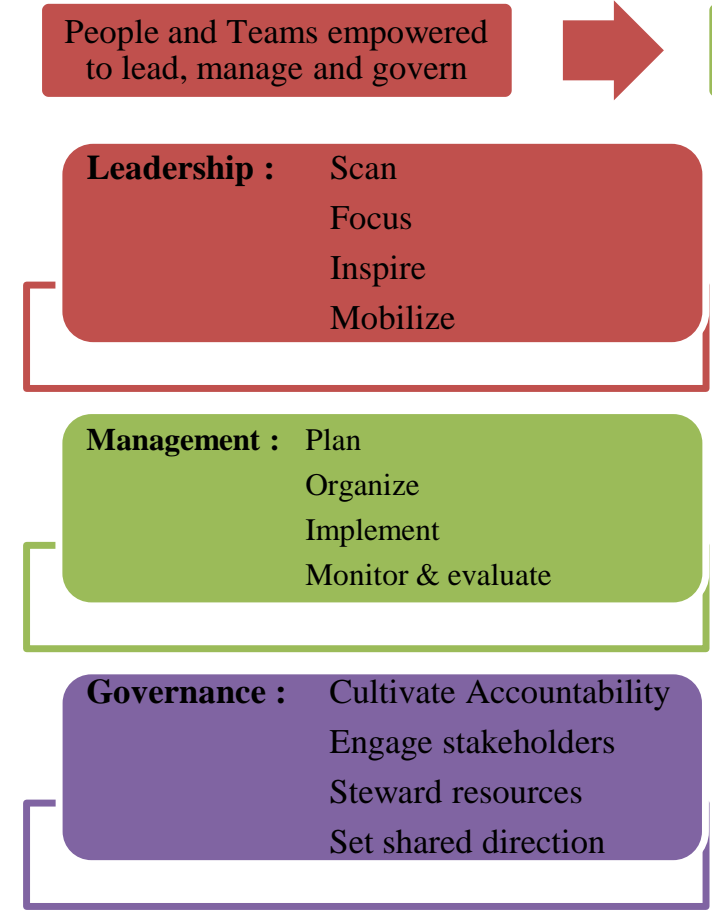

Improved health systems governance
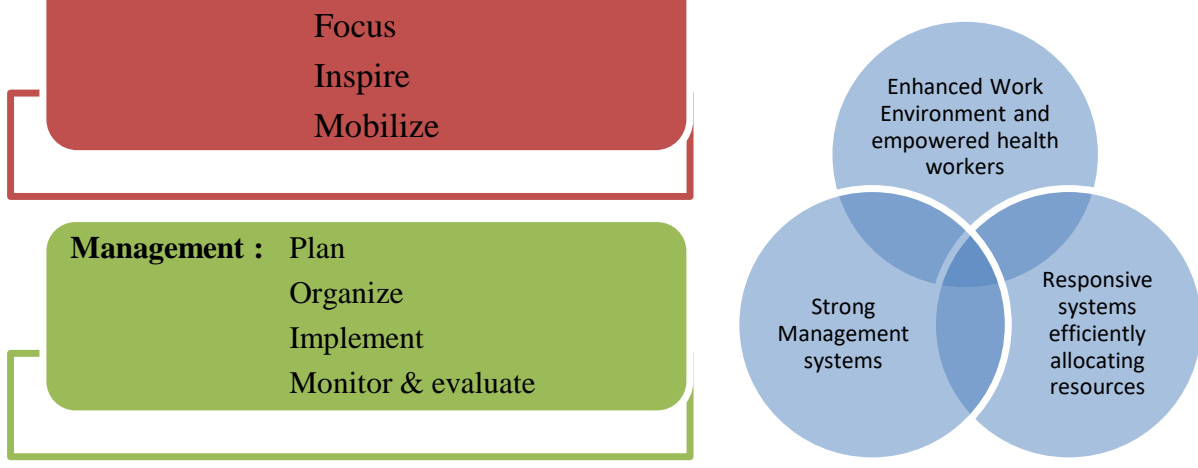
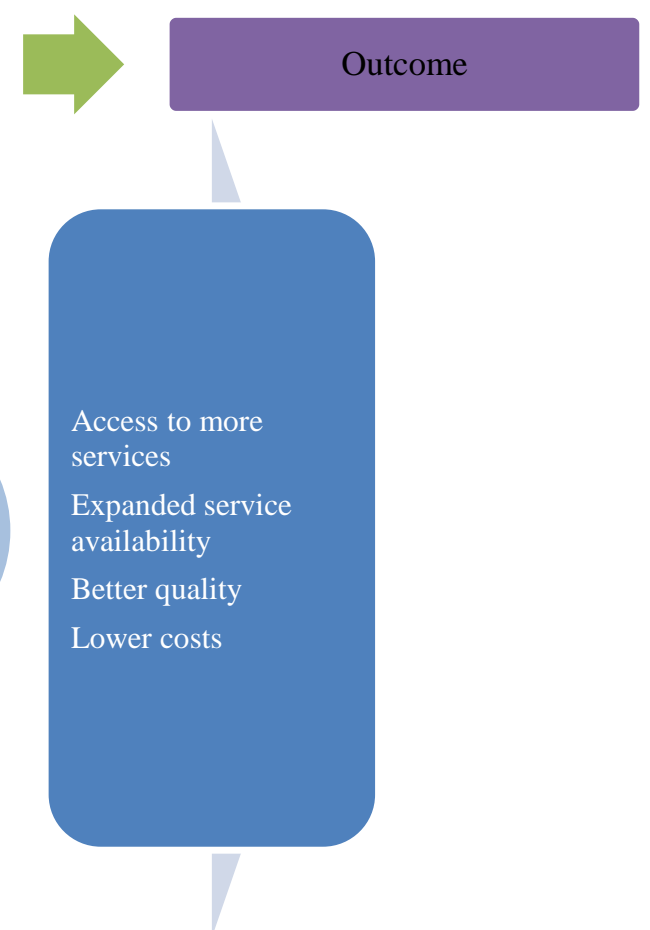

Fig. 1. Conceptual Model of Leadership, Management and Governance (LMG) Practices.

Through the programme, there is development of courses which largely focused on the overview of the health systems, governance in health, leadership in health, management in health, health financing, health service delivery, monitoring and evaluation of health management information systems, ethics, and leadership as well as procurement and supply management in relation to health systems. It also developed specially-tailored online LMGHSS modules for senior policy makers in health (such as health economists), advocacy to increase government resource allocation to LMG-HSS and operations research on health systems leadership, management and governance as well as UHC.

The programme formulated a training program aimed at providing training that integrates key management concepts and best practices and that would equip the senior managers and policy makers with skills in strategic thinking and leadership. This was towards aligning the health sector landscape to economic and population policies, the political and social dynamics as well as offering transformational learning experience that would prepares the senior executives to assume responsibilities at the highest level as they lead their institutions to achieve sustainable competitive edge. The programme also sought to institutionalize the aspects of leadership, management and governance in order to have a sustainable UHC in SSA. This study therefore assessed the experiences, critical success factors and recommendations for improvement of UHC through institutionalizing health system governance in an emerging economy.

Global estimations indicate that close to 150 million people finance their health with out-of-pocket expenditure of which close to 100 million people worldwide are pushed into poverty as a result of high health expenditures (Knickman, \& Kovner, 2015). As a result, UHC is being touted as a panacea. However, healthy systems in SSA remain fragile.

While conceding that the reasons for the poor implementation of universal coverage in emerging economies are complex, others argue that the core cause is a lack of political and technical leadership (Harries et al. 2009). In low-income nations, there is insufficient creative, innovative, decisive, responsible, and responsive, transparent, example, and inspirational leadership to encourage all stakeholders to work together to accomplish the constructive goal of universal coverage (Gonani et al. 2015). Regardless of the current state of these countries' health systems and resources, competent leadership would develop a clear national goal for universal coverage and commit to achieving it gradually over time, thereby measurable progress. As a result, it became necessary to investigate the relationship between health system governance and Universal Health Coverage in a developing country.

Specifically, this study aimed to:

i. Assess the experiences and lessons learnt from PHSSA in improvement of UHC through institutionalizing health system governance in an emerging economy.

ii. Establish the critical success factors for improvement of UHC through institutionalizing health system governance in an emerging economy.

iii. Recommend ways in which UHC can be improved through institutionalizing health system governance in an emerging economy.

\section{Methodology}

A meta-analysis and scan of peer reviewed, gray literature on LMG influence and impact was adopted. In metaanalysis, a review of existing published literature, policy papers and conference papers on leadership and governance 
in health were reviewed, analysed and main themes obtained. A rapid assessment technique led the literature search, which took around six months to complete. Furthermore, the report and conclusions of Amref Health Africa in relation to the PHSSA program's objectives were evaluated. The findings are not intended to be a systematic or exhaustive review of the literature, but rather a formative assessment of the state of the evidence to stimulate debate and inform future research and study in the area of health system governance in the pursuit of Universal Health Coverage in an emerging economy.

\section{FINDINGS}

\section{A. Experiences and Lessons Learnt}

Institutionalizing leadership, management and governance in health systems requires that ministries of health, health professionals, other agencies and stakeholders be empowered to recognize and emphasize leadership in their endeavor of promoting quality universal health care. This implies having the right people in charge; those who have inculcated in them leadership, management and governance skills and knowledge as well as having s organizational arrangements and structures that promote participatory leadership. The adoption of capacity building in key areas of the health system as part of the overall national health sector strategy in a country is key to providing recognition of the LMG-HSS course and institutionalizing it in the long run.

In addition, training institutions such as Medical Training Colleges, Universities as well as other tertiary institutions offering health courses need to integrate the existing aspects of leadership, management, and governance in their curriculum so as to develop whole rounded health professionals who will discharge their duties appropriately. This can be achieved through advocacy by the relevant regulatory bodies that oversee higher education.

Identifying key stakeholders in the health sector, understanding the roles they play in having capacity building initiatives recognized, approved, and accredited is key to institutionalizing the course. The key stakeholders include health regulators, boards, associations, and councils among others. Pursuing partnerships with them and ensuring the course is well recognized is key to building sustainable implementation frameworks.

Faith based institutions and private sector are key to supporting the implementation of capacity building initiatives. A huge percentage of health workforce in a country are from their sectors and once they inculcate LMGHSS into their operational frameworks, they will recognize and include it as part of their continuous development as well as their organizational strategies.

Health Policy interrogation and participating in its review and updates is key to building supporting institutionalization of capacity building programmes in the various countries. Strategies such as making a training a requirement for the health workers or incorporating the need for the health workers to take up the training as either part of their performance review or requirement to completion of the probation into the health employment are some of the key ways of having a programme institutionalized.
It was also noted that since the African population through collaboration with civil societies, NGOs or their political representatives are the primary stakeholders in universal health care consumption, there is a need to empower them to demand for quality health care through participatory leadership. This will create a responsive action not only from the governments but also from the health practitioners and managers. It was also established that health workforce governance and leadership capacity at national and other levels is fundamental to steering the country's HSS agenda to deliver effective health services.

\section{B. Critical Success Factors}

It was established that one of the critical success factors for effective institutionalization of health system governance in an emerging economy is alignment of program objectives and goals to global and country strategies for Health System Strengthening. This will make it easy to market the LMGHSS training to different stakeholders in health. Another critical success factor is clarity of program goals and objectives to all partners and stakeholders involved.

It was also established that commitment of the partners and clear distribution of roles and activities among the stakeholders involved is important in driving the project success. Another factor is availability of a sustainable ownership plan. Such a plan can detail the approaches to building and strengthening of partnerships with stakeholders and beneficiaries; approaches to establishing strong networks and involvement of the project beneficiaries from the start. There is also a need for consolidation and engagement of a strong pool of trainers and delivery of the training by the same facilitators thus ensuring consistency.

Another critical factor for success was commitment by the programme partners which can contribute to success in the implementation of the programme. Understanding different country contexts, that is culture, education levels, bureaucracy, internet accessibility played an important role in different countries' ability to implement the rollout of the programme activities. Another factor is a sustainable financing plan. Without a proper financial support system institutionalization of leadership, management and governance would have minimal impact on the effectiveness and efficiency of health systems.

\section{CONCLUSION}

Because of the variety and complexity inherent in the health system governance model, the findings from the PHSSA program led to the conclusion that governance links in health systems, as well as the outcomes they produce, are contingent rather than assured. What may be achieved from the design of health governance policies and the investments to implement them is influenced by the situation-specific environment of a country's health system. As a result, a generic method cannot be applied across countries. Another conclusion is that health governance must be understood in the context of the larger set of governance institutions that surround it, as well as the social, political, cultural, and historical terrain in which it operates. 


\section{IMPLICATIONS AND RECOMMENDATIONS FOR POLICY AND PRACTICE}

To successfully institutionalize health system governance, there is a need for the relevant policy makers, stakeholders, and governments to develop a competency framework that would ensure recruitment of competent health managers having appropriate knowledge, attitudes, skills and behaviors in line with their positions, roles and duties. The competence of the managers can also be developed further through mentoring, coaching and action learning as well as ensuring the development of experiential skills and tacit knowledge in the workplace environment.

The countries in emerging economies who seek to institutionalize health system governance are required to develop and support an organizational structure and context that sustains leadership practices through advocacy. This would allow ministries of health across Africa to effectively work across other sectors of government and advocate for health funding and promotion of health matters in all policies. The implication is that this will enable the health ministry to work in various governance arrangements with other donors, national and international and civil societies that have specific expertise to support and supplement the resources of the ministry in its core functions.

There is also a need to create an enabling environment for health systems leadership, management and governance through the development of ethics and other competences specific to universal health care situations. The environment in which health managers work clearly influence their effectiveness. The environment includes immediate working environment like nature of the health management team, support, incentives among others. The wider working environment include other public, private sector and NGOs and their role in health systems. This also includes the broad cultural, political, and economic environments which health systems need to contend with.

Without a proper financial support system institutionalization of leadership, management and governance would have minimal impact on the effectiveness and efficiency of health systems. The financial support systems include funding, planning, financial management, information management as well as management of stocks like drugs and infrastructure. Ensuring support systems exists and works solely depends on the leaders in the health systems. The ministry of health in conjunction with the health system leadership or each working separately should take the lead in identifying and developing financial support systems in partnership with other stakeholders including the general population. The policies and structures that allows and sustains these collaborations should be conceptualized and institutionalized.

Institutionalizing short courses, seminars and conferences in health leadership, management and governance will be crucial to entrench participatory leadership in health systems. These provide an open opportunity for health managers to share knowledge particularly tacit knowledge and experiences on managing health systems. With short courses institutionalized through health professional bodies it is possible for health managers to acquire knowledge and skills necessary to govern health systems. Critically, a framework ought to be put in place to collate and recognize the short courses as counting towards professionalism and a career path in health systems leadership and management.

\section{ETHICS APPROVAL AND CONSENT}

All operations in this study were carried out in line with Amref Health Africa's ethical standards, the National Commission for Science, Technology, and Innovation's ethical standards, as well as the 1964 Helsinki declaration and its later revisions or comparable ethical standards.

\section{CONSENT FOR PUBLICATION}

The authors agree that the information in this manuscript should be considered for publishing by your journal, and that the results in this manuscript have not been published elsewhere or are under consideration by another publisher.

\section{AVAILABILITY OF DATA AND MATERIALS}

This publication includes all of the data collected and analyzed during the survey, as well as a supplementary file. On request, the dataset is also available from the relevant author.

\section{CONFLICT OF INTEREST}

The authors declare that they have no conflict of interest.

\section{FUNDING}

The study was funded by Amref Health Africa together with the Ministry of Health (Kenya) and the Japan International Corporation Agency (JICA).

\section{AUTHORS' CONTRIBUTIONS}

Jennifer Chepkorir conceptualized, designed, performed statistical analysis and participated in the initial draft of the study. Nicholas Kiambi and Brenda Nangehe participated in the design of the study and contributed to the finalization of the manuscript. The manuscript was reviewed by Naphtali Agata with commentaries. All authors read and approved the final manuscript.

\section{ACKNOWLEDGMENTS}

This study and manuscript would not have been possible without the financial support provided by Amref Health Africa together with the Ministry of Health (Kenya) and the Japan International Corporation Agency (JICA).

\section{REFERENCES}

Adams, A. M., Ahmed, S. M., \& Evans, T. G. (2018). Universal health care in Bangladesh-promises and perils. The Lancet Global Health, 6(1), e10-e11.

Alliance for Health Policy and Systems Research (AHPSR) et al.2016. Flagship Report. Open Mindsets. Participatory Leadership for Health. 
European Journal of Business and Management Research www.ejbmr.org

Geneva: World Health Organization.

Amref Health Africa (2011). Training Needs Assessment for Health and Health Related Professionals in Sub-Saharan Africa.

Chigudu, S., Jasseh, M., d'Alessandro, U., Corrah, T., Demba, A., \& Balen, J. (2018). The role of leadership in people-centred health systems: a sub-national study in The Gambia. Health policy and planning, 33(1), e14-e25.

Cutler, D. (2012). Policy initiatives to improve the quality of healthcare under universal health coverage in low-and middle-income countries. the case of Thailand (Doctoral dissertation, Birkbeck, University of London).

Gonani, A., \& Muula, A. S. (2015). Point of View: The importance of Leadership towards universal health coverage in Low Income Countries. Malawi Medical Journal, 27(1), 34-37.

Harries, A. D., Schouten, E. J., Ben-Smith, A., Zachariah, R., Phiri, S., Sangala, W. O., \& Jahn, A. (2009). Health leadership in sub-Saharan Africa.

Kieny, M. P., \& Evans, D. B. (2013). Universal health coverage. Eastern Mediterranean Health Journal, 19(4), 305.

Knickman, J. R., \& Kovner, A. R. (2015). The Future of Health Care Delivery and Health Policy. Jonas and Kovner's Health Care Delivery in the United States, 333.

Lagomarsino, G., Garabrant, A., Adyas, A., Muga, R., \& Otoo, N. (2012). Moving towards universal health coverage: health insurance reforms in nine developing countries in Africa and Asia. The Lancet, 380(9845), 933-943.

Ministry of Health (2015). Report of the training needs assessment of Kenya's health workforce, http://www.health.go.ke.

Singh, P., \& Sachs, J. D. (2013). 1 million community health workers in sub-Saharan Africa by 2015. The Lancet, 382(9889), 363-365.

Unger, J. P., Marchal, B., \& Green, A. (2003). Quality standards for health care delivery and management in publicly oriented health services. The International journal of health planning and management, 18(S1), S79-S88.

World Health Organization. (2007). Everybody's Business: Strengthening Health Systems to Improve Health Outcomes. WHO's framework for action. WHO, Geneva.

World Health Organization. (2010). World health statistics 2016: monitoring health for the SDGs sustainable development goals. World Health Organization.

World Health Organization. (2016). World health statistics 2016 monitoring health for the SDGs sustainable development goals. World Health Organization.

World Health Organization. (2008). The world Health Report: Primary Health Care: Now more than ever. Geneva: World Health Organization.

Zaidi, S., Khan, A. L., Mukhi, A. A., Memon, Y., Slama, S., \& Jabbour, S. (2014). Integration of non-communicable diseases into primary health care: a snapshot from Eastern Mediterranean region. 